\title{
475 GEN-011-101 (THE TITAN-1 TRIAL): PHASE 1 STUDY TO \\ EVALUATE THE SAFETY, PROLIFERATION AND PERSISTENCE OF GEN-011, AN AUTOLOGOUS NEOANTIGEN-TARGETED PERIPHERAL T CELL THERAPY IN SOLID TUMORS
}

Thomas Davis*, Arthur DeCillis, Richard Hernandez, Jessica Price, Craig Carey, Kevin Mancini. Genocea, Centreville, MD, USA

Background GEN-011 is a personalized neoantigen-targeted peripheral blood T cell therapy (NPT) developed for the treatment of adult patients (pts) with solid tumors. The proprietary ATLAS $^{\mathrm{TM}}$ (Antigen Lead Acquisition System) will be used to identify true immunogenic neoantigens from each patient's tumor mutanome that are recognized by their own CD4+ and/or CD8 + T cells. ATLAS will also identify Inhibigens ${ }^{\mathrm{TM}}$, antigen targets of $\mathrm{T}$ cells that promote tumor growth. ${ }^{1}$ Autologous peripheral $\mathrm{T}$ cells will be specifically stimulated by up to 30 ATLAS-identified neoantigens, avoiding Inhibigens, to generate an adoptive $\mathrm{T}$ cell product. Preliminary data show yields of billions of highly active T cells with $96 \%$ neoantigen targeting across $89 \%$ of ATLAS selected neoantigens.

Methods TITAN-1 is a multicenter Phase 1 study of GEN-011 NPTs in patients with refractory melanoma, non-small cell lung cancer (NSCLC), squamous cell carcinoma of the head and neck (SCCHN), urothelial carcinoma (UC), renal cell carcinoma (RCC), small cell lung cancer (SCLC), cutaneous squamous cell carcinoma (CSCC), and anal squamous cell carcinoma (ASCC). Patients may enter into one of 2 cohorts of 6 DLT-evaluable patients, either a multiple lower dose (MLD) regimen of GEN-011 as an IV infusion at 4-week intervals, up to 5 doses maximum without lymphodepletion, or a single high dose (SHD) regimen of GEN-011 after flu/cy lymphodepletion. Each dose of GEN-011 will be followed by a course of interleukin-2 (IL-2). Patients will be followed for safety, immunogenicity, and anti-tumor activity over approximately a 5-month treatment period. A long-term follow-up will continue through 2 years after the initial dose of GEN011.

Trial Registration ClinicalTrials.gov identifier: NCT04596033

\section{REFERENCES}

1. Lam $\mathrm{H}$, et al. An empirical antigen selection method identifies neoantigens that either elicit broad anti-tumor response or drive tumor growth. Cancer Discovery 2021 March; 11(3):696-713.

Ethics Approval This study was approved by Western Institutional Review Board, approval number 1-1078861-1

http://dx.doi.org/10.1136/jitc-2021-SITC2021.475 